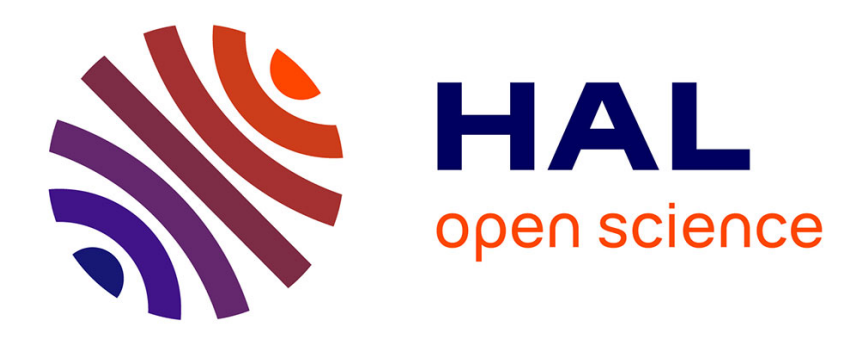

\title{
Design and control of an active anti-roll system for a fast rover
}

\author{
Mohamed Krid, Faïz Ben Amar
}

\section{To cite this version:}

Mohamed Krid, Faïz Ben Amar. Design and control of an active anti-roll system for a fast rover. 2011 IEEE/RSJ International Conference on Intelligent Robots and Systems, 2011, San Francisco, United States. pp.274-279. hal-03177947

\section{HAL Id: hal-03177947 https://hal.science/hal-03177947}

Submitted on 23 Mar 2021

HAL is a multi-disciplinary open access archive for the deposit and dissemination of scientific research documents, whether they are published or not. The documents may come from teaching and research institutions in France or abroad, or from public or private research centers.
L'archive ouverte pluridisciplinaire HAL, est destinée au dépôt et à la diffusion de documents scientifiques de niveau recherche, publiés ou non, émanant des établissements d'enseignement et de recherche français ou étrangers, des laboratoires publics ou privés. 


\title{
Design and control of an active anti-roll system for a fast rover
}

\author{
Mohamed Krid and Faiz Benamar
}

\begin{abstract}
Off-road operational conditions require large suspension displacements and a significant clearance between the ground and the main frame, yielding to an elevated position of the vehicle mass center. Consequently, this makes the vehicle more likely to turn over when cornering fast. This paper proposes a new design, and its associated control, of an active device which improves the stability of fast rover moving up to $10 \mathrm{~m} / \mathrm{s}$. The proposed design can be equipped on any offroad chassis which has independent suspensions. We propose the using of an active anti-roll system allowing the control of the roll angle and thus improving the vehicle stability, especially when turning or when moving on slopping ground. The proposed system increases the controllability of the vehicle, by giving access to the roll angle which is usually uncontrollable. We develop a model based predictive controller for the roll dynamics, which minimizes the load transfer during cornering and the energy consumed by the actuators. The control model is based on a dynamic model of the rover and on a stability criteria defined by the lateral load transfer. Dynamic simulation, carried out for different rover trajectories with different speeds, show the benefit of the proposed active system and the validity of the control approach.
\end{abstract}

\section{INTRODUCTION}

Outdoor mobile robots has to perform operations more and more far and more and more quickly. In this way, we are interesting on the design and control of fast rovers which are able to move autonomously in natural environment following, at high velocity (up to $10 \mathrm{~m} / \mathrm{s}$ ), a virtual corridor referred by GPS points. Generally, off-road rovers have an elevated center of gravity for rock collision avoidance and have an important suspension displacement for obstacle crossing. These two features combined to a high speed could cause tip-over situations, generally along the lateral direction. Moreover, actual perception systems are not sufficiently accurate and rapid to carry out a safe autonomous land crossing. Indeed, the robot could undergo important disturbances due to collision with obstacles or to the contact failures when crossing holes. These disturbances can create instabilities, or even rover tip-over. Hence, it is interesting to develop active mechanical systems which make possible system state correction when it approaches these hazardous situations. In this context, our work introduces a new solution based on the use of an active anti-roll mechanism, which can avoid these situations and increases the stability margin of the vehicle, thus improving the mobility and crossing performance of the robot.

This work was supported by FAST project (Fast AutonoumouS allTerrain robot) of ANR-PSIROB program

ISIR - Institut des Systèmes Intelligents et Robotiques Université Pierre et Marie Curie Paris 6, CNRS UMR 7222 4, place Jussieu - 75252 Paris Cedex 05 - France krideisir. upmc. fr amardisir.upmc. fr
Stability control is a central issue for developing safe navigation on natural terrain. Nowadays, this is done by introducing in the trajectory generator appropriate constraints characterizing the tip-over conditions. [1] considers a stability domain defined in the 2D-space velocity-path curvature and applied to a rigid robot model. [2] considers a complex car model including a tire relationships to define a model predictive control which optimizes over a receding horizon a quadratic criteria composed by the trajectory parameters and the control inputs. This method, and in similar manner [3][4], utilize wheel torques and/or steering angles as control inputs. This stability control approach limiting the system velocity and/or the steering angle can lead to a conservative system, thus reducing the system performance. The second approach for vehicle stabilization consists in integrating a dedicated mechanical device. In this paper, we will focus mainly on the stabilization along the roll direction and on the minimization of the lateral tip-over risk.

Anti-roll systems have been widely studied and developed in automotive industry. The passive anti-roll bar which links the left and right wheels, is deformed when right and left suspensions are moving oppositely. Generally, this bar has a $U$ form and is already integrated in major part of cars. However, this linkage introduce a coupling between suspensions, and can transfer a perturbation from one side to the other one, for example when a single wheel crosses over a bump or a hole.

Numerous solutions had been already proposed to equip vehicles with stability active control system [5]. Among these, the solution based on an active anti-roll bar seems to be the most studied [6][7]. This one, inspired from the passive anti-roll device, use a hydraulic actuator in place of the torsion bar. This actuator can create an opposite displacement between left and right suspensions but it does not participate when these displacements are equal (with the same sign). Hydraulic actuation is chosen for its high-power, robustness and the faculty of inhibition (bypass mode interconnecting the two chambers of each cylinder). Also we mention the concept of inter-connected suspensions whose principle consists in connecting the hydraulic dampers in many ways by using distributors [8]. Independent active suspension are using hydraulic cylinders placed in parallel with the conventional shock absorbers [9] [10], however a position-based control of this actuator can hardly provide a compliant behavior with adequate time response. Another original idea developed by Bose consists in replacing the shock absorbers by linear electromagnetic actuators [11]. 
This paper presents an innovative mechatronic device which is able to control the roll stability of the rover. Firstly, we start by presenting the kinematics of the mechanical system and its velocity and force transmission. The third section presents the dynamic model of the vehicle, and especially its roll dynamics and the stability index based on the lateral load transfer. Based on the linearized form of this dynamic model, a model based predictive control is designed. The control assumes that the desired path and velocity are known over the receding time horizon. The control optimizes a mixed criteria which combines the stability of the robot and its power consumption. Finally, we present simulation results carried out for different trajectories of the robot. Simulations are done using Matlab-Simulink/Adams co-simulation.

\section{ACTIVE ANTI-ROLL MECHANISM}

Nowadays, most of on-road vehicles are equipped with passive anti-roll bar. Thanks to a flexible bar which couples the right and left suspensions, the roll angle is minimized when the vehicle performs a cornering. This system goes against any asymmetrical movements between right and left suspensions, and especially when the vehicle is submitted to a lateral acceleration (due to centrifugal or gravitational forces). The proposed mechanism, inspired from this passive system, replaces the torsion bar by an actuator which by creating opposite motion between right and left suspensions, changes the roll configuration of the rover (fig.1). The horizontal linear actuator when changing its length, it acts on two rocking-levers which create two opposite deformations on the right and left double-wishbone suspensions (2).

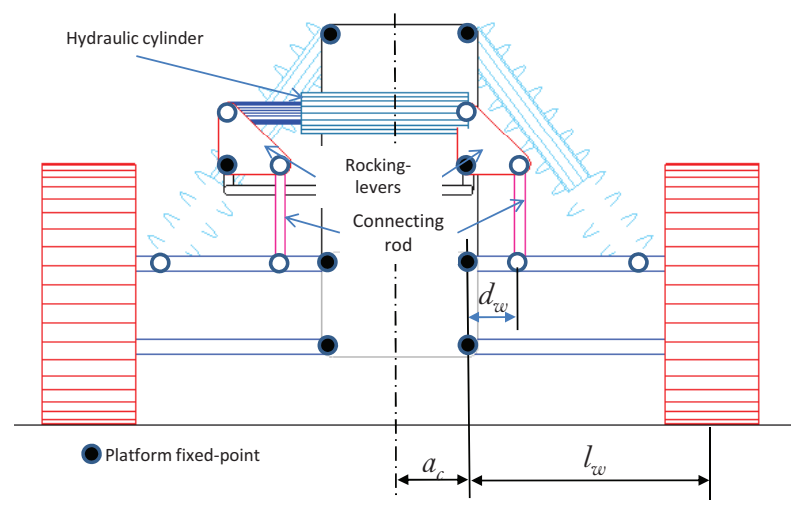

Fig. 1. Kinematics of the active anti-roll mechanism.

It is clear that this system create a coupling between suspensions and can decrease the crossing capacity of unilateral discontinuities. However, the hydraulic actuation can be made quasi-transparent by interconnecting the two chambers of each cylinders. Thus by using hydraulic distributors and bypass cylinders, we can inhibit this anti-roll system, functions of the ground conditions and others robot parameters. Considering power and design constraints, this system is identically reproduced on the two front and rear axles. Modeling this system with Adams software, this design appears to be quite linear in the interval of $\pm 15 \mathrm{deg}$ with respect to the cylinder displacement (3). This plot, achieved with a periodic sinusoidal motion imposed on the cylinder, looks to have "some hysteresis". This is due wheel-ground contact compliance and dynamics effects.

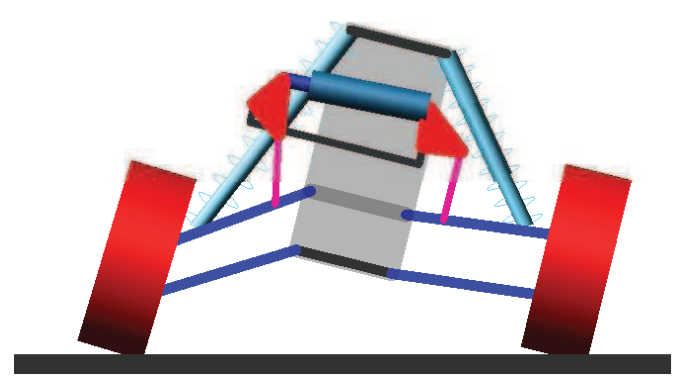

Fig. 2. Adams model view.

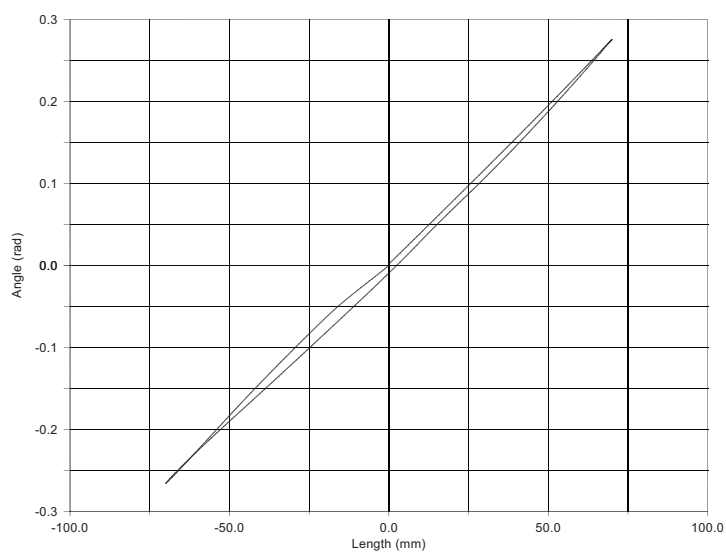

Fig. 3. Roll angle (rad) as function of the actuator displacement (mm).

Despite the asymmetry of the fixation point positions of the rocking-levers, this relationship is symmetrical. In fact, the roll motion is achieved thanks to the difference between vertical displacements of the connecting rods. The roll center for a double wishbone-suspension is located for the nominal configuration at the ground surface and in the medium plane. When the platform tilts the position of the mass center of the chassis moves along the lateral direction and thus changing the contact load distribution. Figure (4) shows the locations of instantaneous rotation centers $I_{i j}$ of the double wishbones suspension mechanism. $I_{i j}$ depicts the rotation point of body $i$ with respect to body $j$. Planar rigid body motion properties mention that $I_{i j}, I_{j k}, I_{k i}$ are collinear. Using this features, $I_{w g}$ can be determined at the intersection of lines $\left(I_{c g}, I_{c w}\right)$ and $\left(I_{w s}, I_{s g}\right)$. Approximating this four-bars mechanism by a parallelogram, the angular velocity of the stub axle with 
respect to the ground can be considered equal to that of chassis $\dot{\phi}$. Then, the absolute velocity of points $I_{w c}$ and $I_{s w}$ can be determined as function of $\dot{\phi}$ and the geometrical dimensions $l_{w}, a_{c}$ defined on figure (1). Then the angular velocity with respect to ground of the upper wishbone can be quite easily demonstrated to be equal to $-\frac{a_{c}}{l_{w}} \dot{\phi}$, and thus its angular velocity with respect to the chassis is $\left(-\frac{a_{w}}{l_{c}}-1\right) \dot{\phi}$. Consequently, the magnitude of the linear velocity with respect to the chassis of the connecting rod can be given by $d_{w}\left(\frac{a_{c}}{l_{w}}+1\right) \dot{\phi}$. Finally, the ratio between the cylinder displacement $\delta \lambda$ and the roll angle $\delta \phi$ can be given by

$$
\frac{\delta \lambda}{\delta \phi} \approx 2 d_{w}\left(\frac{a_{c}}{l_{w}}+1\right)=k_{m}
$$

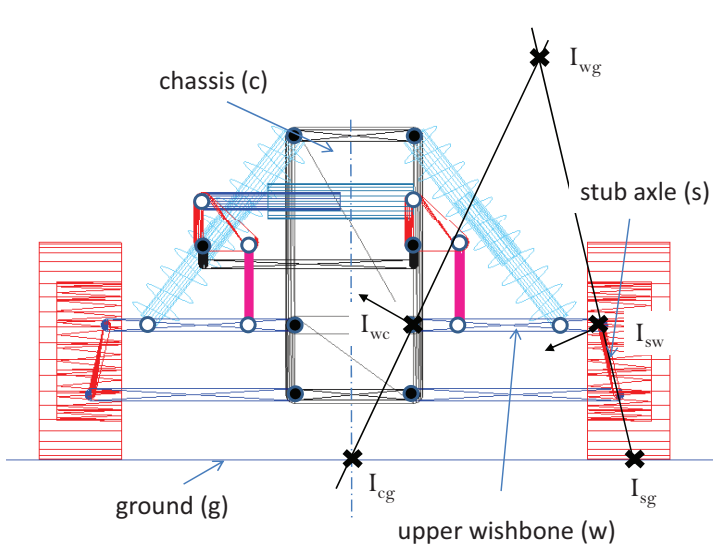

Fig. 4. Location of instantaneous rotation centers.

For $a_{c}=130, l_{w}=450, d_{w}=100 \mathrm{~mm}$, the latter relationship gives a mechanism ratio $k_{m}=258 \mathrm{~mm} / \mathrm{rd}$, which is quite close to the "exact" value $(275 \mathrm{~mm} / \mathrm{rd})$ computed by Adams (fig.3). This relationship gives main dimensions which are relevant for the design of this device, especially the distance $d_{w}$ is determinant for sizing the cylinder stroke and the required thrust.

\section{VEHICLE MODEL}

The goal of this part is to build a model for the control of the anti-roll device and to provide a measure for stability margin. A vehicle is a multibody system with a complex kinematic architecture and with a complex interaction with the ground. However, the number of degrees of freedom of the model can be reduced for achieving a simplified characterization of the vehicle dynamics. The dynamics of each wheel suspension (unsprung mass) will not be considered in this model but an equivalent 1 dof model of the suspension will be used for representing the roll motion of the sprung mass [12] [13]. In the model presented in this paper, only the planar dynamic and the roll (fig.5 and 6). First, we will remind the model of the vehicle without the anti-roll system.
In this model, the effect of pitching is neglected. The steering angle $\beta_{f}$, the actuator torque on each wheel $C_{m i}$ and the braking torque $C_{f i}$ forms the inputs parameters of the navigation control. The side slip angles of the vehicle are neglected. Let's define the state variables : $V_{x}, V_{y}$ for the longitudinal and lateral velocities, $V_{\psi}$ for the yaw rate of the vehicle, $\phi$ for the roll angle of the sprung mass and $\omega_{i}$ for the rate of wheel $i$. $M$ depicts the total vehicle mass, $I_{i j}$ are its inertia tensor components, $M_{s}$ the sprung mass, $h_{p}$ the height of the center of mass with respect to the roll center (see previous section), $k_{\phi}$ and $c_{\phi}$ are respectively the roll stiffness and damping. These latter are related to stiffness and damping of the spring-dampers by linear relationships depending on the geometrical dimensions of the suspension mechanism.

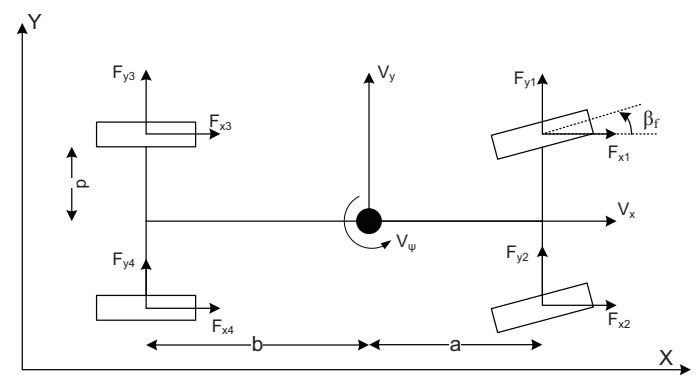

Fig. 5. A four wheeled vehicle with front steering.

The vehicle dynamics depends on gravity, the aerodynamic forces and mainly on ground forces produced by tires. The interaction forces between the wheel and the ground are generally described by the semi-empirical Pacejka model [14] [15]. If the contact forces are projected on the reference frame of the wheel like expressed in model (1), we obtain a nonlinear system. The lateral forces $F_{y i}$ and the longitudinal ones $F_{x i}$ appear in all equations of model (1) except for the fourth one describing the roll dynamics of the sprung mass. This one will be used for designing a control model of the anti-roll system. If we neglect the term $I_{x z} \ddot{\psi}$ in the roll dynamics equation, we can express the fourth equation of the model (1) as follow

$$
\begin{aligned}
\dot{x}_{\phi}= & \left(\begin{array}{cc}
0 & 1 \\
a_{21} & a_{22}
\end{array}\right) x_{\phi} \\
& +\frac{1}{I_{x s}}\left(\begin{array}{c}
0 \\
M_{s} g h_{p}\left(\dot{V}_{y}+\dot{\psi} V_{x}\right)
\end{array}\right)
\end{aligned}
$$

$$
\text { with } x_{\phi}=\left(\begin{array}{c}
\phi \\
\dot{\phi}
\end{array}\right) \text { state and } a_{21}=\left(M_{s} g h_{p}-2 k_{\phi}\right) / I_{x s}
$$

The second term of equation (3) depends on the lateral dynamics and mainly the centrifugal force $M_{s} \dot{\psi} V_{x}$ which is given directly by the vehicle horizontal trajectory. This nonlinear part will determine the equilibrium point of the roll angle associated with the null-control. 


$$
\begin{aligned}
\dot{V}_{x} M= & M \dot{\psi} V_{y}-M_{s} h_{p} \dot{\psi} \dot{\phi}+\left(F_{x 1}+F_{x 2}\right) \cos \beta_{f}+\left(F_{x 3}+F_{x 4}\right) \cos \beta_{r}-\left(F_{y 1}+F_{y 2}\right) \sin \beta_{f}-\left(F_{y 3}+F_{y 4}\right) \sin \beta_{r} \\
\dot{V}_{y} M= & M_{s} h_{p} \ddot{\phi}-M \dot{\psi} V_{x}+\left(F_{x 1}+F_{x 2}\right) \sin \beta_{f}+\left(F_{x 3}+F_{x 4}\right) \sin \beta_{r}+\left(F_{y 1}+F_{y 2}\right) \cos \beta_{f}+\left(F_{y 3}+F_{y 4}\right) \cos \beta_{r} \\
\ddot{\psi} I_{z}= & I_{x z} \ddot{\phi}+a\left(\left(F_{x 1}+F_{x 2}\right) \sin \beta_{f}+\left(F_{y 1}+F_{y 2}\right) \cos \beta_{f}\right)+d\left(\left(F_{x 2}-F_{x 1}\right) \cos \beta_{f}+\left(F_{y 1}-F_{y 2}\right) \sin \beta_{f}\right) \\
& -b\left(\left(F_{x 3}+F_{x 4}\right) \sin \beta_{r}+\left(F_{y 3}+F_{y 4}\right) \cos \beta_{r}\right)+d\left(\left(F_{x 4}-F_{x 3}\right) \cos \beta_{r}+\left(F_{y 3}-F_{y 4}\right) \sin \beta_{r}\right) \\
\ddot{\phi} I_{x s}= & M_{s} h_{p}\left(\dot{V}_{y}+\dot{\psi} V_{x}\right)+I_{x z} \ddot{\psi}+M_{s} g h_{p} \phi-2\left(k_{\phi} \phi+c_{\phi} \dot{\phi}\right) \\
I_{\omega} \dot{\omega}_{i}= & C_{m i}-C_{f i}-R_{e} F_{x i}, i=1, \ldots, 4 .
\end{aligned}
$$

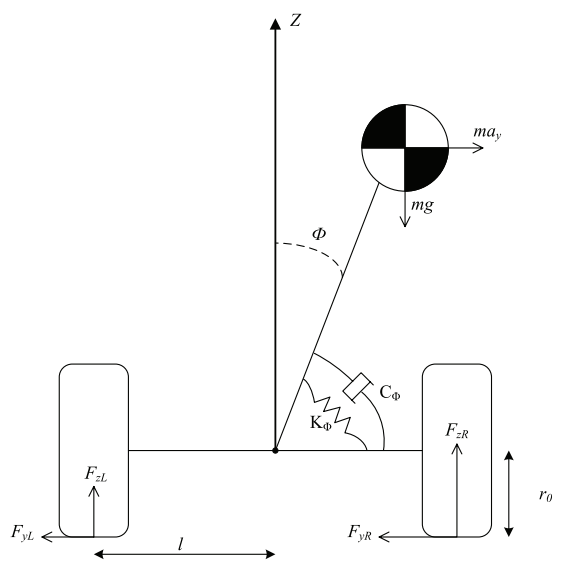

Fig. 6. Vehicle model along the roll direction.

If we consider now the anti-roll system introduced in the previous section, clearly it does not change the system parametrization. Then, we can consider a new torque $u_{\phi}$ due to the cylinder force $F_{a}$ as a new generalized force associated to the roll parameter $\phi$ which will be added to other terms of equation (3). Applying the principle of virtual work or the kineto-static duality in multibody rigid systems, we can write

$$
u_{\phi}=\frac{\delta \lambda}{\delta \phi} F_{a}=k_{m} F_{a}
$$

where $k_{m}$ is the mechanism constant defined in the previous section. Then the roll dynamics model can be rewritten as follows

$$
\begin{aligned}
\dot{x}_{\phi}= & \left(\begin{array}{cc}
0 & 1 \\
a_{21} & a_{22}
\end{array}\right) x_{\phi}+\left(\begin{array}{c}
0 \\
1 / I_{x s}
\end{array}\right) u_{\phi} \\
& +\frac{1}{I_{x s}}\left(\begin{array}{c}
0 \\
M_{s} g h_{p}\left(\dot{V}_{y}+\dot{\psi} V_{x}\right)
\end{array}\right)
\end{aligned}
$$

\section{Controller Design}

The goal of the control of the anti-roll system would be the minimization of tip-over risks, or the maximization of a stability margin. Numerous stability margin was already defined, going from simple static one based on the minimal distance of the mass center vertical projection to the boundaries of the convex hull support to complex dynamic measure based on ZMP. However in our dynamic quasiplanar problem, the load lateral transfer (LLT) between right and left wheels can be used as a measure of the tip-over risk [16]. The main objective of the proposed control will be to stabilize the vehicle by reducing the lateral load transfer (LLT). It is demonstrated in [6] that

$$
L L T=\left|\Delta F_{z}\right|=\left|\frac{M_{s} g h_{p} b}{(a+b) d} \phi+\frac{M h}{d} a_{y}\right|
$$

where $a_{y}$ is the lateral acceleration of the vehicle mass center, $h$ its height with respect to ground surface. Solving the equation $L L T=0$, we obtain a reference roll angle $\phi_{\text {ref }}$,

$$
\phi_{\text {ref }}=-\frac{M h(a+b)}{M_{s} g h_{p} b} a_{y}(t)
$$

which minimizes the lateral load transfer (6).

As it can be seen in this latter expression, $\phi_{\text {ref }}$ depends on the lateral acceleration $a_{y}(t)$. Thus it is necessary to replace the lateral acceleration $a_{y}(t)$ by $a_{y_{r e f}}$. This latter can be computed easily from the reference robot trajectory. In our case the reference path and the desired speed are supposed to be known at this control level. If we neglect the side slip angle of the vehicle mass center, we can determine $a_{y_{r e f}}$ from the path curvature $\kappa_{\text {ref }}(t)$ and the forward velocity $V_{\text {xref }}(t)$, by the following relationship

$$
a_{y_{r e f}}(t)=-k_{r e f}(t) V_{x \text { ref }}^{2}
$$

Then, using this latter equation, equation (7) becomes

$$
\phi_{\text {ref }}(t)=\frac{M h(a+b)}{M_{s} g h_{p} b} k_{r e f}(t) V_{x r e f}^{2}
$$

This reference angle is bounded to the extremal values of the roll angle determined directly by the cylinder stroke. In order to take into account the dynamic constraints and to anticipate future set point changes, a model-based predictive control MPC method is used. We start first by splitting the control input into two terms, i.e.

$$
u_{\phi}=u_{\phi l}+u_{\phi c}
$$

where

$$
u_{\phi l}=-M_{s} g h_{p}\left(\dot{V}_{y}+\dot{\psi} V_{x}\right)
$$


a linear decoupling term, that can be estimated from the robot on-board sensors. Thus the non-linear model (3) takes now the following linear form

$$
\dot{x}_{\phi}=A_{\phi} x_{\phi}+B_{\phi} u_{\phi c}
$$

Let consider at the instant $t$ the objective function $\mathrm{J}$ defined by :

$J\left(x_{\phi}(t), \Delta U(t)\right)=\sum_{i=t+1}^{t+p}\left\|\phi_{i, t}-\phi_{r e f_{i, t}}\right\|_{Q}^{2}+\sum_{i=t}^{t+c-1}\left\|\Delta u_{i, t}\right\|_{R}^{2}$

which represents a weighted sum of the reference tracking errors of $\phi_{i, t}$ (the predicted value of $\phi$ at time $i$ computed at instant $t$ ) and of control input changes $\Delta U(t)=$ $\left[\Delta u_{i, t}, \ldots, \Delta u_{t+c-1, t}\right]$. These latter components are defined by

$$
u_{i+1, t}=u_{i, t}+\Delta u_{i, t}
$$

$Q$ and $R$ are weighting matrices and $p$ and $c(\leq p)$ are prediction time horizon and control time horizon. After the resolution of the quadratic optimization problem (13), only the first term of $\Delta U(t)$, is used to compute the control inputs $u_{\phi c, t}$ of the linearized model that must be applied to the system, that is to say

$$
u_{\phi c, t}=u_{\phi c, t-1}+\Delta u_{t, t}
$$

This roll controller can be considered as decoupled from that dedicated for path tracking (fig.7). The path controller unit manages the trajectory tracking by adjusting the speed and the steering angles that minimize the error between the reference trajectory and the actual position of the robot. The roll controller acts directly on the anti-roll actuator to enhance vehicle stability by minimizing the roll angle and the lateral load transfer (LLT).

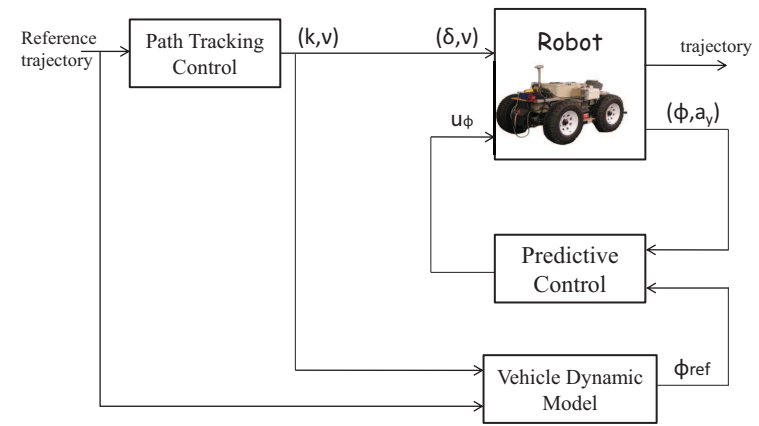

Fig. 7. Architecture of the roll control of an autonomous robot.

\section{Simulation RESUlts}

The MPC controller presented in the previous section has been tested using Matlab-Simulink/ADAMS co-simulation. Adams software computes the vehicle multi body dynamics, which includes a wheel-ground contact model. The Adams model is a four-wheeled chassis with independent doublewishbone suspensions and with 2 anti-roll mechanism integrated on each axle. The contact model includes wheel's stiffness and damping, and a regularized Coulomb friction law parametrized by static and dynamic friction coefficients. Matlab-Simulink reads Adams output (i.e. the roll angle), computes the optimal control input (i.e. the actuator force) and send it to Adams.

The goal of the simulation tests is to quantify the benefits of the anti-roll device, especially when the vehicle performs a cornering manoeuvre. First, we will present simulation results for a circular path obtained by constant control inputs $\beta_{f}=12^{\circ}$ for the front steering angle and $V_{x}=5 \mathrm{~m} / \mathrm{s}$ for the forward velocity. The circular path starts from instant $t=3 \mathrm{~s}$, and is preceded by a straight-line path for speed rise phase.

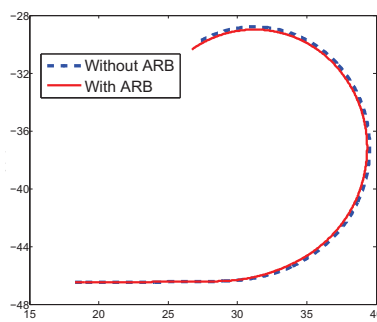

(a)

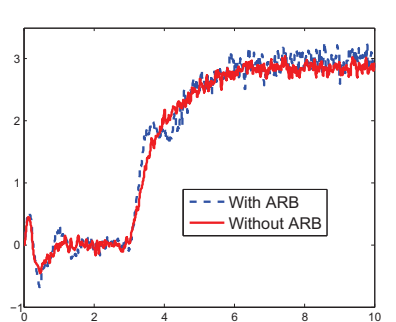

(b)
Fig. 8. (a) Robot center path, (b) lateral acceleration as function of time

Curves of figure (8) show the robot trajectories and the lateral accelerations in both cases, with and without antiroll device. By comparing these curves, we can conclude the possibility of neglecting the anti-roll effect on the horizontal robot motion and the availability of path/posture decoupling. This allows us to integrate the anti-roll device without changing the path control signals.

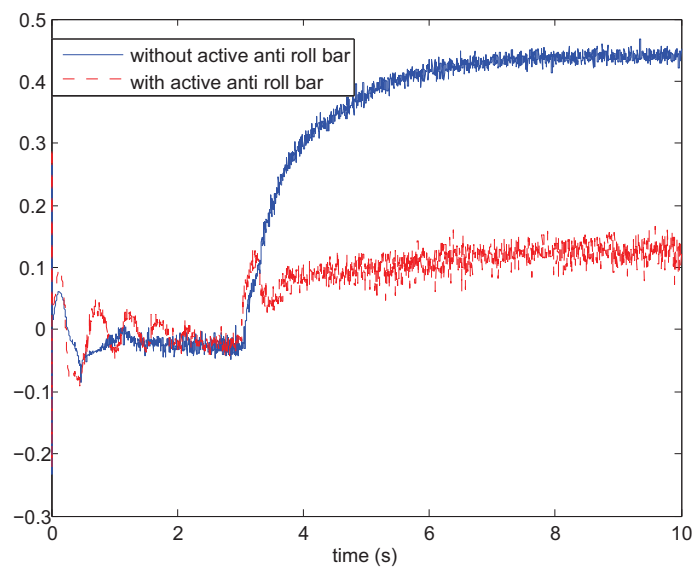

Fig. 9. Normalized Lateral Load Transfer $L L T$ as function of time.

In figure (9), we give the Normalized Lateral Load Transfer NLLT as function of time for both cases : with and without anti-roll control. Normalization is done by dividing LLT by the total weight. Thanks to the anti-roll control, the load transfer is minimized $N L L T \approx 0.1$ regarding the 
passive case where $N L L T=0.45$. Curves oscillations are mainly due to the high eigenfrequency introduced by the contact stiffness of the Adams contact model.

For this test, we give also on figure (10) the roll angle and the control input i.e. the actuator force. We can see that the roll control provides a roll behavior opposite to the natural one (without control). This behavior looks to that of moto racer who leans into the turn for centrifugal force compensation.

The benefits of the active anti-roll can be more demonstrated when increasing the velocity displacement $V_{x}$. Figure (11) shows the lateral load transfer as function of the vehicle speed and with a constant steering angle. By comparing the vehicle's LLT with and without the active roll device, we can clearly notice the benefits of this system on the tip-over stability. For the uncontrolled roll case, the robot exhibits a instability when the speed reaches $7 \mathrm{~m} / \mathrm{s}$, in this case the $N L L T$ exceeds the limit of 1 . However, the anti-roll control increases the stability margin of the robot as the LLT is minimized and opens the possibility to the robot to travel with a speed beyond $7 \mathrm{~m} / \mathrm{s}$ with a sufficient security margin (NLLT remains below 0.5).

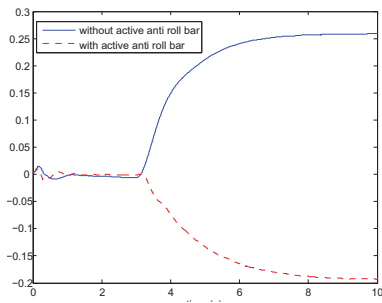

(a)

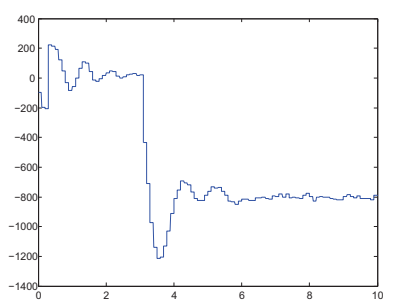

(b)
Fig. 10. (a) Roll angle, (b) Actuator force as function of time.

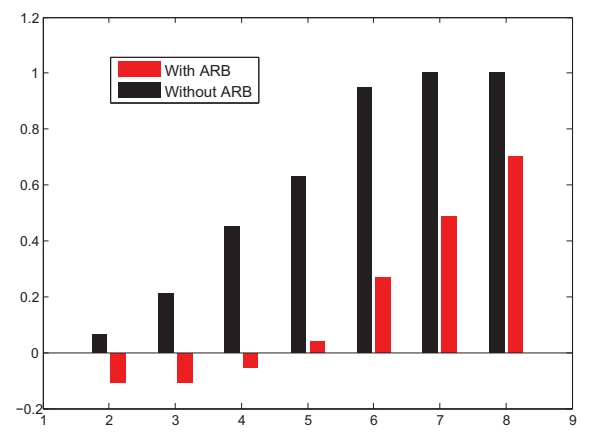

Fig. 11. Normalized Lateral Load Transfer as function of robot speed.

\section{CONCLUSION}

In this paper an integrated approach of an active anti-roll system has been presented. An innovative kinematics which can be easily added on existent off-road chassis is proposed. A model predictive controller based on minimization of load transfer and energy consumption is designed. Simulation results show that this system improves the performance and the stability of the robot when cornering. An important advantage of the proposed solution is its easy integration as new part, without any transformation of the original chassis. This system can be controlled independently and is demonstrated to have not effect on the dynamics of path controller. A new rover based on an existent commercial chassis is currently under construction to equip it with electric actuators, sensors, and the active anti-roll system detailed in this paper. The next steps, will focus on the experimental validation of this promising new system. Another challenge for increasing offroad performance, would be the design and development of innovative systems for preventing tip-over instability along both roll and pitch axes.

\section{REFERENCES}

[1] M. Spenko, Y. Kuroda, S. Dubowsky, and Iagnemma K. Hazard avoidance for high-speed mobile robots in rough terrain. Journal of Field Robotics, 23 (5) :311-331, 2006

[2] G. Palmieri, P. Falcone, H.E. Tseng, and L. Glielmo. A preliminary study on the effects of roll dynamics in predictive vehicle stability control. In Decision and Control, 2008. CDC 2008. 47th IEEE Conference on, pages 5354 -5359, 2008.

[3] C. Zhao, W. Xiang, and P. Richardson. Vehicle lateral control and yaw stability control through differential braking. In Industrial Electronics, 2006 IEEE International Symposium on, volume 1, pages $384-389$, 2006.

[4] Y.B Seo, J.W. Choi, and G.R. Duan. Lateral vehicle control using the ccv mode control. In Control Conference, 2006. CCC 2006. Chinese, pages $41-46,2006$.

[5] Y. Shiao, C. Lai, and Q. Nguyen. The analysis of a semi-active suspension system. In SICE Annual Conference 2010, Proceedings of, pages $2070-2082,2010$.

[6] D.J. Sampson. Active Roll Control of Articulated Heavy Vehicles. Engineering department, Cambridge University Engineering Department, September September 2000.

[7] A.J.P. Miège. Active Roll Control of an Experimental Articulated Vehicle. PhD thesis, Cambridge University Engineering Department, October Octobre 2003.

[8] P.H. Cronjé. Improving off-road vehicle handling using an active anti roll bar. Master's thesis, University of Pretoria, 2008.

[9] S. Zhou and S. Zhang. Semi-active control on leaf spring suspension based on smc. In Control and Decision Conference (CCDC), 2010 Chinese, pages 1462 -1466, May 2010.

[10] F. Kou and Z. Fang. An experimental investigation into the design of vehicle fuzzy active suspension. In Automation and Logistics, 2007 IEEE International Conference on, pages 959 -963, 2007.

[11] http ://www.bose.com.

[12] Y. Abbassi. Contribution à la modélisation et à la commande de la dynamique du véhicule. $\mathrm{PhD}$ thesis, Université de Technologie BelfortMontbéliard / Université de Franche-Comté, 2007.

[13] J.Y. Wong. Terramechanics and off-road Vehicles. Elsevier, 1989.

[14] H.B. Pacejka. Tyre and vehicle dynamics. Butterworth-Heinemann, 2002.

[15] C. Liu, Y. Meng, J. Zhang, and C. Kong. Dynamics simulation for 4ws vehicle steering performance. In Advanced Computer Theory and Engineering (ICACTE), 2010 3rd International Conference on, volume 5, pages V5-108 -V5-110, 2010.

[16] N. Bouton, R. Lenain, B. Thuilot, and J.-C. Fauroux. A rollover indicator based on the prediction of the load transfer in presence of sliding : application to an all terrain vehicle. In Robotics and Automation, 2007 IEEE International Conference on, pages 1158 1163, 2007. 